\title{
Subretinal Fibrosis in Stargardt's Disease with Fundus Flavimaculatus and ABCA4 Gene Mutation
}

\author{
Settimio Rossi Francesco Testa Marcella Attanasio \\ Ada Orrico Antonella de Benedictis Michele Della Corte \\ Francesca Simonelli \\ Department of Ophthalmology, Second University of Naples, Naples, Italy
}

\section{Key Words}

Stargardt's disease $\cdot$ ABCA4 gene $\cdot$ Subretinal fibrosis $\cdot$ Ocular trauma $\cdot$ Lifestyle

\begin{abstract}
Purpose: To report on 4 patients affected by Stargardt's disease (STGD) with fundus flavimaculatus (FFM) and ABCA4 gene mutation associated with subretinal fibrosis.

Methods: Four patients with a diagnosis of STGD were clinically examined. All 4 cases underwent a full ophthalmologic evaluation, including best-corrected visual acuity measured by the Snellen visual chart, biomicroscopic examination, fundus examination, fundus photography, electroretinogram, microperimetry, optical coherence tomography and fundus autofluorescence. All patients were subsequently screened for ABCA4 gene mutations, identified by microarray genotyping and confirmed by conventional DNA sequencing of the relevant exons.
\end{abstract}

Results: In all 4 patients, ophthalmologic exam showed areas of subretinal fibrosis in different retinal sectors. In only 1 case, these lesions were correlated to an ocular trauma as confirmed by biomicroscopic examination of the anterior segment that showed a nuclear cataract dislocated to the superior site and vitreous opacities along the lens capsule. The other patients reported a lifestyle characterized by competitive sport activities. The performed instrumental diagnostic investigations confirmed the diagnosis of STGD with FFM in all patients. Moreover, in all 4 affected individuals, mutations in the ABCA4 gene were found.

Conclusions: Patients with the diagnosis of STGD associated with FFM can show atypical fundus findings. We report on 4 patients affected by STGD with ABCA4 gene mutation associated with subretinal fibrosis. Our findings suggest that this phenomenon can be accelerated by ocular trauma and also by ocular microtrauma caused by sport activities, highlighting that lifestyle can play a role in the onset of these lesions. 


\section{Introduction}

Juvenile macular degeneration was first reported in 1909 by the German ophthalmologist Karl Stargardt from whom the disease gets its name. Stargardt's disease (STGD) is a bilateral, symmetrical and progressive macular dystrophy characterized by a decrease in central vision and the presence of bilateral atrophicappearing foveal lesions. These lesions may have a beaten metal appearance and are associated with yellowish-white fundus flecks at the posterior pole and/or midperipheral retina [1].

An angiographic characteristic sign is the dark choroid, also known as choroidal silence, that probably results from the accumulation of abnormal material in the retinal pigment epithelium (RPE), presumably lipofuscin [2]. Fishman and Lois classified STGD into 4 stages [3, 4]. Stage 1 includes patients with parafoveal or perifoveal flecks. Normal results were most frequently obtained on electroretinogram (ERG; Lois I). Lois et al. [4] have described a reduced-cone ERG in some patients at this stage. Patients with flecks appearing throughout the posterior pole, anterior to the vascular arcades and/or nasal to the optic disc, are classified as stage 2. ERG amplitudes were most often normal, but subnormal cone and rod responses may be observed (Lois II). Stage 3 includes patients with extensive flecks that have resorbed almost entirely. Subnormal cone or cone and rod ERG amplitudes were often reported (Lois III). Stage 4 includes patients with extensive atrophy of the RPE and choroids. ERG testing shows notably reduced cone and rod amplitudes (Lois IV). According to this classification, patients with partially resorbed extensive flecks are classified as stage 2-3.

Genetically, STGD is a heterogeneous disorder that is usually inherited in an autosomal recessive manner and can rarely present as an autosomal dominant trait. In 1994, STGD was mapped to the long arm of chromosome 1 and was later shown to be caused by mutations in the $A B C A 4$ (formerly $A B C R$ ) gene.

The pathogenesis of the disease is related to mutations in the ABCA4 gene which encodes an ATP-binding cassette (ABC) transport protein located in the disc membranes of rod and cone outer segments $[5,6]$.

The ABCA4 protein is involved in the transport of all trans-retinal conjugates across the disc membranes. In the absence of a functional ABCA4 protein due to mutations in the gene, vitamin A aldehyde forms bisretinoid products that are deposited in RPE cells during the process of disc shedding and phagocytosis. Consequently, abnormally high levels of lipofuscin pigments, such as A2E and the all trans-retinal dimer phosphatidylethanolamine, accumulate in the RPE, triggering RPE cell death and causing secondary photoreceptor degeneration $[7,8]$.

Atypical fundus findings have been described, including pallor of the optic disc, attenuated retinal vessels, retinal pigmentation in the form of bone spicule, chorioretinitis scar, retinal pigment hyperplasia, and subretinal neovascularization $[9,10]$.

Furthermore, atypical fundus alterations identified as areas of subretinal fibrosis have been observed in patients affected by STGD. In the literature, there are few articles that report on cases with STGD associated with subretinal fibrosis. In only 1 case, these lesions were not associated with a trauma, and none of the cases reported genetic analysis for the ABCA4 gene [9-15]. 
Here, we report the presence of subretinal fibrosis in 4 patients affected by STGD with fundus flavimaculatus (FFM) and ABCA4 gene mutations.

\section{Patients and Methods}

Four patients with subretinal fibrosis were ascertained through the Retinal Research Center of the Eye Clinic of the Second University of Naples, Italy, from a pool of 384 patients with a clinical diagnosis of STGD [16]. Research procedures were in accordance with the institutional guidelines and the Declaration of Helsinki. Informed consent was obtained from all patients after having explained the nature of the procedures to be performed.

All 4 patients underwent complete eye examinations, including medical and ocular history of the patient and his or her family, visual acuity, fluorescein angiography, ERG, optical coherence tomography (OCT), microperimetry (MP), and fundus autofluorescence (FA). Medical and ocular history was analyzed thoroughly, highlighting any head/ocular trauma reported or sport activity practiced by each patient. Best-corrected visual acuity (BCVA) was examined with the Snellen visual chart, full-field electroretinography was recorded by means of a corneal contact lens electrode with Ganzfield stimulator according to international clinical standards (ISCEV). Based on ophthalmoscopic findings and electrophysiological tests, all STGD patients were classified into 4 stages according to the Fishman and Lois classification [3, 4].

FA was performed with confocal scanning laser ophthalmoscopy (cSLO Heidelberg Retina Angiograph 2; Heidelberg Engineering Dossenheim, Germany). The study has been carried out using fundus photography with an excitation filter of $448 \mathrm{~nm}$ and a barrier filter of $495 \mathrm{~nm}$. OCT was performed with SD-OCT (Cirrus HD OCT; Carl Zeiss, Dublin, Calif., USA). MP is a testing modality that can be used to measure and map central retinal sensitivity in macular diseases. The MP-1 microperimeter (Nidek, Padua, Italy) is a commercially available device that performs fundus tracking and automated image alignment for eye movements, enabling a precise and repeatable mapping of retinal sensitivities of discrete points in the macula.

\section{Mutation Detection}

All 50 exons of the $A B C A 4$ gene were screened for mutations by a combination of single-strand conformation polymorphism (SSCP) and heteroduplex analysis in all affected patients. In all cases in which a pattern different from the wild-type SSCP was identified, the corresponding exon was sequenced. Segregation analysis and screening of controls were performed by SSCP analysis under optimized conditions.

\section{Results}

\section{Case 1}

Case 1 is a 35-year-old female who was diagnosed with STGD at the age of 6 years, with a heterozygous mutation in the ABCA4 gene (N965S/G1961E) which was confirmed by genetic analysis in 2004 .

In her clinical history, the patient reported an ocular trauma in the right eye, which required hospitalization and caused sudden loss of vision at the age of 9 years. In 1998, at our first observation, visual acuity was 20/1,000 in the right eye and 20/600 in the left eye.

Biomicroscopic examination of the anterior segment showed a nuclear opacity of lens dislocated to the superior site as well as vitreous opacities along the posterior capsule lens in the right eye. Examination of the anterior segment of the left eye did not show any pathologic alterations. Fundus examination revealed an area of subretinal fibrosis associated with diffuse pigmentation in both eyes (ig. 1a, fig. 2). ERG showed 
extinguished scotopic and photopic responses (Lois IV), and retinal fluorangiography reported a degree of hypofluorescence corresponding to flecks and dark choroid. These unusual ocular changes in the manifestation of STGD are probably due to ocular trauma. In fact, a previous ERG, performed in 1984, showed a normal response. In 2009, the patient underwent MP, OCT and FA exam. MP revealed instable fixation in both eyes with no detectable retinal mean sensitivity $(0 \mathrm{~dB})$.

Finally, FA pointed out the localization of the peculiar area of fibrosis in the left eye. These diagnostic exams could not be performed in the right eye because of a nuclear cataract dislocated in the superior site after ocular trauma. At present time, visual acuity remains unchanged.

\section{Case 2}

Case 2 is a 20 -year-old male who was diagnosed with STGD at the age of 11 years, with a homozygous mutation in the ABCA4 gene (c.571-2A>T intron 5) which was confirmed by genetic analysis in 2009 .

The patient came to our observation in 2006 with a visual acuity of 20/32 in the right eye and 20/50 in the left eye. He did not report any ocular trauma in his clinical history but his lifestyle was characterized by competitive sport activities: tennis, football from the age of 6 to 14 years and later swimming at least twice a week up to the present.

Biomicroscopic examination of the anterior segment showed a healthy ocular adnexa, and specular, transparent and 'in situ' lens that may justify the absence of any ocular trauma as reported by the patient's clinical history.

Fundus examination demonstrated the presence of variably pigmented changes in the macula associated with flecks that extended beyond the vascular arcades (Fishman II), and an area of subretinal fibrosis associated with diffuse pigmentation in the superior juxta-papillar region of the right eye (fig. 1b, fig. 2). ERG presented subnormal scotopic and photopic responses (Lois II). MP showed instable fixation in both eyes with low retinal mean sensitivity ( $7.4 \mathrm{~dB}$ in the right eye to $8.6 \mathrm{~dB}$ in the left eye).

Finally, FA pointed out a hypofluorescent central area corresponding to macular atrophy and hypofluorescent areas corresponding to flecks. After 3 years, a new ophthalmological examination showed small atrophic areas, some with pigment, localized in the temporal sector of the left eye. In 2012, these lesions have become areas of subretinal fibrosis localized in an inferior-temporal sector of the midperipheral retina. Visual acuity has gradually reduced. At present, BCVA is 20/200 in both eyes.

\section{Case 3}

Case 3 is a 21-year-old male who was diagnosed with STGD at the age of 10, with heterozygous mutations in the ABCA4 gene (S1071L; K223Q; G1203R) which were confirmed by genetic analysis in 2009 .

The patient did not report any ocular trauma in his clinical history but his lifestyle was characterized by competitive sport activities: he played rugby from the age of 7 to 17 at least twice a week. He came to our observation in 2009 with a BCVA of 20/200 in both eyes. 
Biomicroscopic examination of the anterior segment showed healthy ocular adnexa, and specular, transparent and 'in situ' lens that may justify the absence of any ocular trauma reported by the patient's clinical history.

Fundus examination showed the presence of variable pigmentary changes in the macula associated with flecks that extended beyond the vascular arcades (Fishman II) and a small inferior juxta-papillar area of subretinal fibrosis associated with pigment in the right eye, while in the left eye this lesion was localized in the posterior pole, macula temporally (fig. 1c, fig. 2). ERG showed normal scotopic and photopic responses (Lois I). MP reported instable fixation in the right eye and stable fixation in the left eye with low retinal mean sensitivity in both eyes $(8.0 \mathrm{~dB}$ in the right eye and $10.0 \mathrm{~dB}$ in the left eye). Visual acuity remained stable.

\section{Case 4}

Case 4 is a 34-year-old female who was diagnosed with STGD at the age of 10 years. Genetic analysis in 1998 allowed the identification of one mutation in the ABCA4 gene (A1038V). The patient did not report any ocular trauma in her clinical history and her lifestyle was characterized by competitive sport activities, in particular she played volleyball from the age of 10 to 18 twice a week. She came to our observation in 1998 with a BCVA of 20/100 in both eyes.

Biomicroscopic examination of the anterior segment did not show any signs of ocular trauma as confirmed by the clinical history reported by the patient. An ophthalmologic exam reported bilateral atrophic-appearing foveal lesions associated with yellowish-white fundus flecks at the posterior pole (Fishman I). ERG showed subnormal scotopic and photopic responses (Lois II).

In 2001, after 3 years, a new ophthalmologic examination showed an area of subretinal fibrosis in the midperiphery of the left eye (fig. 1d, fig. 2). Visual acuity was $20 / 240$ in both eyes. The patient underwent retinal fluorangiography that showed a degree of hypofluorescence corresponding to flecks and dark choroid; in the left eye there were hypofluorescent areas due to hyperplasia of RPE and hyperfluorescent areas corresponding to an initial subretinal fibrosis localized in the superior temporal site of the retina.

\section{Discussion}

Our results confirm that patients affected by STGD-FFM can develop areas of subretinal fibrosis. Up to now, only few articles reporting on patients affected by STGDFFM and areas of subretinal fibrosis have been published.

In 1991, Ko et al. [11] reported histologic evidence that a trauma can play a role in releasing massive amounts of lipofuscin from the RPE, suggesting that liberation of lipofuscin might be caused by an overload of photoreceptor debris to the RPE at the time of the trauma. This article described patients with ophthalmologic examination characterized by RPE dystrophy in the macular region but without clinical diagnosis of STGD. 
In 1993, Del Buey et al. [12] reported a case of a 13-year-old girl affected by FFM who developed a fibrotic reaction of the retina and pigmentary migration after a mild ocular trauma in the right eye. They suggested that the damaged RPE could explain the fibroglial reaction of the retina and pigment migration that occurred as a non-specific consequence of an inflammatory process. In 1995, De Laey et al. [9] reported on 3 patients with the characteristics of STGD who developed massive subretinal fibrosis in the fundus. In only 1 case, these lesions were associated to ocular trauma. In 1997, Ober et al. [14] reported a case of a 14-year-old boy affected by STGD who developed an area of subretinal fibrosis after mild ocular trauma. All previous articles presented in the literature described patients affected by STGD but in none of them genetic analysis had been performed.

In our study, all 4 patients present areas of subretinal fibrosis localized in different retinal sectors; however, the extent and severity of these lesions are variable. Our first case (case 1) can support the etiopathogenetic hypothesis of Del Buey et al. [12] about the presence of subretinal fibrosis in patients with STGD-FFM. In case of trauma, the formation of fibrosis is probably faster, more extensive and is associated with a sudden loss of vision. The other 3 cases (cases 2-4) of patients affected by STGD-FFM associated with subretinal fibrosis lesions have no clinical history of ocular trauma, and the formation of fibrosis is gradual and associated with a progressive loss of vision. However, these patients reported a lifestyle characterized by competitive sport activities. Minor ocular trauma or vitreous continuous stress related to sport activities can probably cause changes in the vitreous retinal interface resulting in the damage of RPE that induces liberation of lipofuscin that leads to fibrosis.

Furthermore, all 4 patients present mutations in the ABCA4 gene. The presence of mutations in this gene confirms the diagnosis of STGD, even if the areas of subretinal fibrosis had hidden ophthalmoscopic signs of disease as in case 1.

The mutations identified are missense mutations in 3 cases and a splice-site mutation in 1 case; a correlation between types of mutation and severity of clinical manifestation has been observed. In fact, in accordance with our previous studies [16], missense mutations are associated with mild clinical and electrophysiological exam, while splice-site mutations are correlated to severe forms. In fact, the last 2 cases had been classified as Fishman I, Lois I-II, whereas case 2 had been included in a Fishman II, Lois II classification.

We have also observed that in 3 cases the mutation is expressed in a heterozygous manner (cases 1, 3 and 4) and in 1 case in a homozygous manner (case 2). In fact, 2 of the 3 patients (cases 3 and 4) with a heterozygous mutation in the ABCA4 gene presented small and delimited areas of subretinal fibrosis at an ophthalmologic examination, whereas the patient with a homozygous mutation in the ABCA4 gene (case 2) showed an extended retinal lesion, similar to case 1 who reported ocular trauma in her clinical history.

In conclusion, our observation supports the hypothesis that patients with a diagnosis of STGD can develop subretinal fibrosis as a consequence of damage to the RPE and that this phenomenon can be accelerated by ocular trauma. Moreover, the lifestyle of patients affected by STGD can play a significant role in the onset and 
progression of these lesions. In fact, sport activities can cause minor ocular traumas or vitreous continuous stress determining RPE damage.

Our study highlights the importance of lifestyle in patients affected by STGD and encourages ophthalmologists to inform patients about possible complications induced by sport activities. In addition, our study represents the first article in the literature that describes cases of patients affected by STGD-FFM with subretinal fibrosis and mutations in the ABCA4 gene, suggesting a possible correlation between types of mutations and ophthalmologic manifestations of subretinal fibrosis lesions.
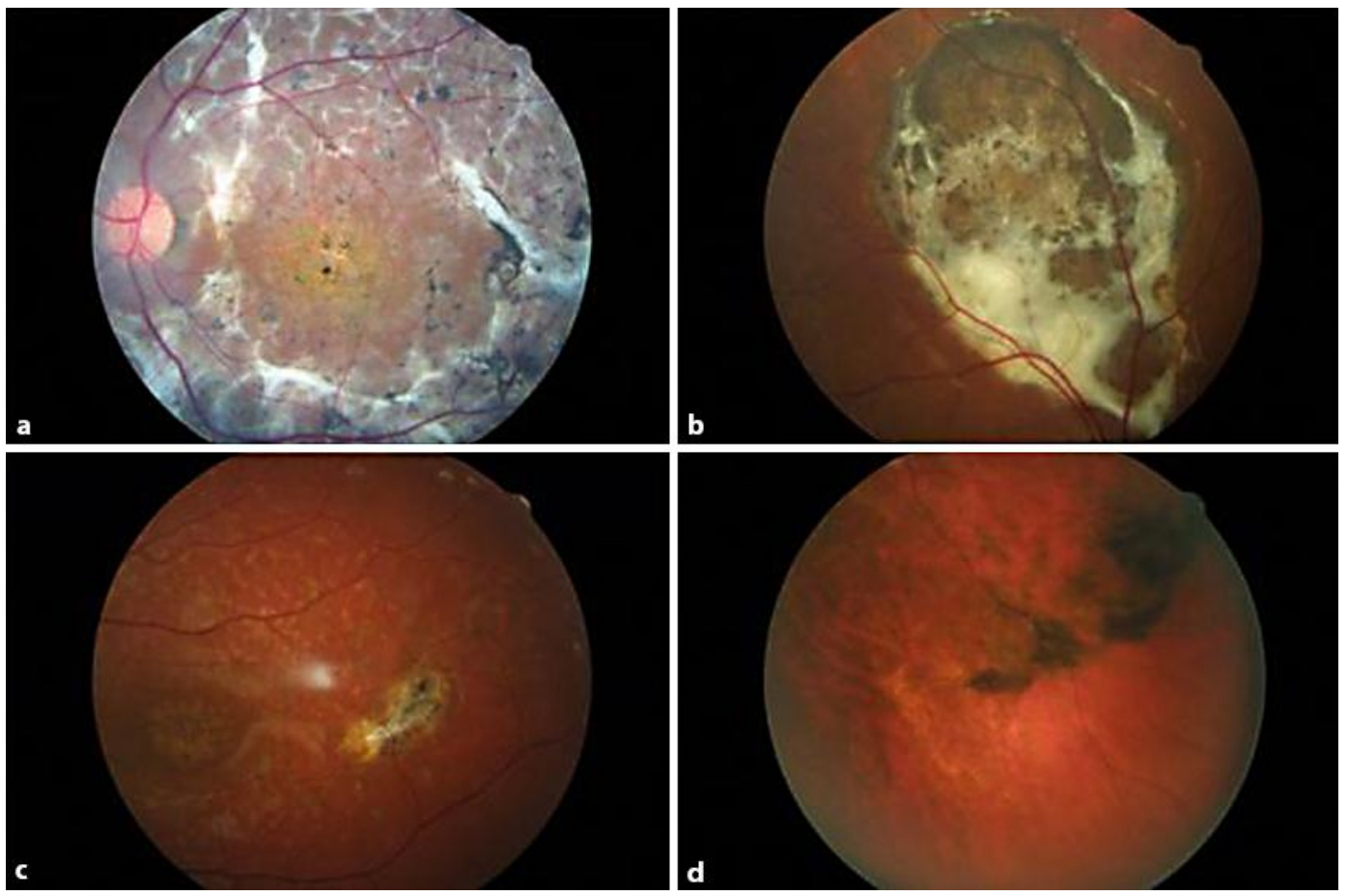

Fig. 1. a Case 1 - left eye: diffuse area of subretinal fibrosis. b Case 2 - right eye: area of subretinal fibrosis in the superior juxta-papillar region associated with diffuse pigmentation. c Case 3 - left eye: small area of subretinal fibrosis, macula temporally. $\mathbf{d}$ Case 4 - left eye: area of subretinal fibrosis in mid-periphery. 


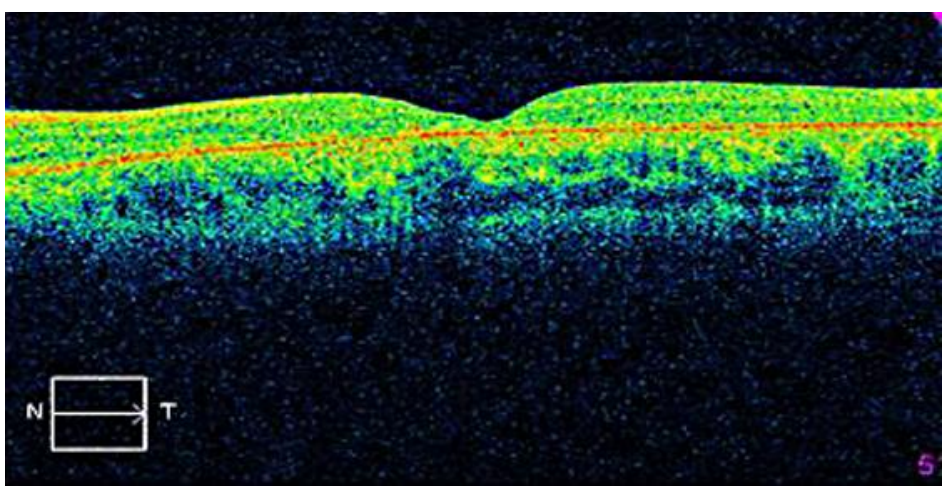

Fig. 2. RPE dystrophy associated with a reduction of macular thickness.

\section{References}

1 Stargardt K: Über familiäre, progressive Degeneration in der Makulagegend des Auges. Albrecht von Graefes Arch Klin Ophthalmol 1909;71:534-550.

2 Fishman GA, Farber M, Patel BS, Derlacki DJ: Visual acuity loss in patients with Stargardt's macular dystrophy. Ophthalmology 1987;94:2061-2067.

-3 Genead MA, Fishman GA, Stone EM, Allikments R: The natural history of Stargardt disease with specific sequence mutation in the ABCA4 gene. Invest Ophthalmol Vis Sci 2009;50:5867-5871.

-4 Lois N, Holder GE, Bunce C, Fitzke FW, Bird AC: Phenotypic subtypes of Stargardt macular dystrophyfundus flavimaculatus. Arch Ophthalmol 2001;119:359-369.

5 Kaplan J, Gerber S, Larget-Piet D, et al: A gene for Stargardt's disease (fundus flavimaculatus) maps to the short arm of chromosome 1. Nat Genet 1993;5:308-311.

6 Gerber S, Rozet JM, Bonneau D, et al: A gene for late-onset fundus flavimaculatus with macular dystrophy maps to chromosome 1p13. Am J Hum Genet 1995;56:396-399.

7 Higgins CF: ABC transporter: from microorganism to man. Annun Rev Cell Biol 1992;8:67-113.

-8 Allikmets R, Singh N, Shroyer NF, Hutchinson A, Chidambaram A, Gerrard B, Baird L, Stauffer D, Peiffer A, Rattner A, Smallwood P, Li Y, Anderson KL, Lewis RA, Nathans J, Leppert M, Dean M, Lupski JR: A photoreceptor cell-specific ATP-binding transporter gene (ABCR) is mutated in recessive Stargardt macular dystrophy. Nat Gen 1997;15:236-246.

-9 De Laey JJ, Verougstraete C: Hyperlipofuscinosis and subretinal fibrosis in Stargardt's disease. Retina 1995;15:399-406.

10 Parodi MB: Progressive subretinal fibrosis in fundus flavimaculatus. Acta Ophthalmol (Copenh) 1994;72:260-264.

11 Ko MK, Lee WR, Mc Kechnie NM, Hall-Parker B: Post-traumatic hyperlipofuscinosis in the human retinal pigment epithelium. Br J Ophthalmol 1991;75:54-60.

12 Del Buey MA, Huerva V, Minguez E, Cristobal JA, Iturbe F, Palomar A: Posttraumatic reaction in a case of fundus flavimaculatus with atrophic macular degeneration. Ann Ophthalmol 1993;25:219-221.

13 Gass JD, Hummer J: Focal retinal pigment epithelial dysplasia associated with fundus flavimaculatus. Retina 1999;19:297-301.

14 Ober RR, Limstrom SA, Simon RM: Traumatic retinopathy in Stargardt's disease. Retina 1997;17:251254

15 Grandinetti AA, Portella E, Arana J, Iskorostenski NT: Subretinal fibrosis in Stargardt's disease: case report. Arq Bras Oftalmol 2011;74:449-451.

-16 Testa F, Rossi S, Passerini I, Di Iorio V, Della Corte M, Banfi S, Surace EM, Menchini U, Auricchio A, Simonelli F: Correlation between photoreceptor layer integrity and visual function in patients with Stargardt disease: implications for gene therapy. Invest Ophthalmol Vis Sci 2012;53:4409-4415.

Settimio Rossi and Francesco Testa contributed equally to this work. 